\title{
Mathematical Modeling of The Transmission Dynamics of Amoebiasis With Some Interventions
}

Stephen Edward Mwaijande ( $\nabla$ stephenmwaihuti@gmail.com )

University of Dodoma

Godfrey Edward Mpogolo

Tanzania Institute of Accountancy

Research Article

Keywords: Amoebiasis, screening, intervention, diarrhea.

Posted Date: March 3rd, 2022

DOI: https://doi.org/10.21203/rs.3.rs-1363033/v1

License: (c) (i) This work is licensed under a Creative Commons Attribution 4.0 International License.

Read Full License 


\title{
Mathematical Modeling of the Transmission Dynamics of Amoebiasis with some Interventions
}

\author{
Stephen Edward Mwaijande ${ }^{1^{*}}$ and Godfrey Edward Mpogolo ${ }^{2}$ \\ ${ }^{1}$ Department of Mathematics and Statistics, University of Dodoma, \\ Postal address: Box 338, Dodoma, Tanzania \\ ${ }^{2}$ Department of Management Studies, Tanzania Institute of Accountancy, \\ Postal address: Box 9522, Dar es Salaam, Tanzania \\ E-mail:stephenmwaihuti@gmail.com (Corresponding author ${ }^{*}$ )
}

\begin{abstract}
A mathematical model for amoebiasis is developed and presented. The model captures some control interventions such as screening, treatment, and sanitation. The effective reproductive number is computed and is used to analyze the stability of the model system. Sensitivity analysis is used to investigate the parameters that impact the transmission of the disease; as such, it could need more attention to bring the disease to an end. Numerical results show a reduction in infections when at least each of the control measures considered is applied efficiently. Moreover, the findings show that carriers play a potential role in the prevalence of amoebiasis, ignoring them undermines the efforts of containing this epidemic. On the other hand, sensitivity analysis shows that indirect transmission contributes to more infections than direct transmission.
\end{abstract}

Keywords: Amoebiasis, screening, intervention, diarrhea.

\section{Introduction}

Amoebiasis is an intestinal disease caused by the unicellular protozoan Entamoeba histolyt$i c a$ and characterized by wet and bloody feces mingled with a mass intestinal mucus called amoebic diarrhea. It is a widespread human gastrointestinal parasitic disease responsible for over 50 million annual infections worldwide and accounts for more than 100,000 deaths annually. Entameoba exists in 3 morphologically identical forms of strains, namely the virulent pathogenic, Entamoeba histolytica, and the two non-pathogenic forms, Entamoeba dispar and Entamoeba Moshkovskii (Haque and Petri 1999; Clark and Diamond 1991; Stauffer and Ravdin 2003; Ximnez et.al 2009) [1, 2, 3, 4]. Amoebiasis is still considered a major public health problem worldwide in tropical and subtropical regions with crowded population, poor hygiene, and lower socioeconomic levels( Fathi et al. 2017)[5].

Moreover, Entamoeba histolytica can evade and harm other internal human organs such as the brain, lungs, and liver. Entamoeba histolytica is also counted among the group of chronic, disabling and disfiguring diseases; commonly called the Neglected Tropical Diseases (NTDs) (Saha et.al 2016 [6].

Most relevant studies used to build our current study are not based on mathematical models except (Saha et al. 2016; Hategekimana et al. 2016; Hategekimana et.al 2017) $[6,7,8])$. They are based on clinical, medical, and other fields related to Entamoeba histolytica. 
Hategekimana et al. 2016 [7] developed a mathematical model that was an SEIR with the addition of carrier-class. This work stems from the first mathematical models developed for amoebiasis until then. Their model has some shortcomings in that it did not capture indirect means through which this disease is mainly transmitted. Furthermore, their model did not consider any control measures such as treatment, screening, and sanitation to assess how it could be used to mitigate the epidemic. An assumption on constant population is not valid since the population is dynamic. In addition, disease-related death was ignored in the previous work. Most results were purely theoretical; no validation was conducted in their study. The critical threshold, such as the reproduction number, was not deduced that could be used to analyze the system.

Saha et al. 2016 [6] extended the work by Hategekimana et al. 2016 [7] by performing a detailed mathematical analysis but without further modification of the previous model. Moreover, they employed the model to mimic the Epidemic Message Propagation in a Delay-Tolerant Network (DTN).

Hategekimana et al. 2017 [8] extended the work by Hategekimana et al. 2016 [7] by performing a detailed mathematical analysis of the model. Numerical simulation simulations were also conducted in their study. However, their study did not account for any intervention strategies to control this disease. Moreover, their study neglected the role of the environmental component in the transmission dynamics of amoebiasis.

This study's primary focus is to develop a mathematical model that accounts for the role of direct and indirect transmission dynamics of amoebiasis in the presence of control interventions. We incorporate treatment, screening, and sanitation and study the benefits of such a study. In principle, the current research improves the work done by Hategekimana et al. 2017 [8].

The rest of the paper is organized as follows: Section 2 focuses on model formulation and analysis, whereas Section 3 is based on the effects of control strategies. Next, numerical simulation is presented in section 4, while Section 5 is devoted to sensitivity analysis, and lastly, Section 6 winds up by giving concluding remarks.

\section{Methods}

\section{Model formulation and analysis}

Motivated by the study of Hategekimana et al. 2017 [8], an extension is made on their work by the addition of an extra compartment that caters reservoir of pathogens in the environment. As such, the current model will capture dual transmission pathways; human-tohuman transmission (direct route) and environment-to-human transmission (indirect route). Moreover, the model considered here captures three control interventions: treatment, sanitation, and screening. The total human population is sub-divided into five mutually exclusive epidemiological classes or subgroups: susceptible $S(t)$, Exposed $E(t)$, infectious $I(t)$, carriers $C(t)$, and recovered $R(t)$. Basically, we modify the $S E I R$ model by adding carrier class $C(t)$. Moreover, in order to improve realism, we incorporate an additional compartment, $B(t)$, which represents the reservoir of amoebiasis pathogens in the environment.

Susceptible individuals are assumed to be recruited into the population at a constant rate $\Lambda$. Susceptible individuals may acquire amoebiasis infection following effective direct contact with infectious individuals or carriers at the time-dependent rate $\lambda_{h}(t)$ or after ingesting environmental pathogens from contaminated aquatic reservoirs at the time-dependent rate 
$\lambda_{b}(t)$. Here the term $\lambda_{h}(t)$ represents direct transmission between individuals, and it is modelled by the standard mass action principle, whereas $\lambda_{b}(t)$ represents indirect transmission which is also modelled by standard mass action. The total force of infection is denoted by $\lambda(t)$ and is given by

$$
\lambda(t)=\lambda_{h}(t)+\lambda_{b}(t)
$$

where

$$
\begin{aligned}
& \lambda_{h}(t)=\alpha_{1} I(t)+\alpha_{2} C(t), \\
& \lambda_{b}(t)=\alpha_{3} B(t) .
\end{aligned}
$$

Moreover, $\alpha_{1}$ and $\alpha_{2}$ are the transmission rates for infectious and carriers individuals respectively while, $\alpha_{3}>0$ is the ingestion rate of Amoebiasis pathogens by individuals. Exposed individuals may either join infectious or carrier classes. A fraction $f$ of the exposed progress to infectious stage at the rate $\delta$ while the compliment become carrier at the same rate $\delta$. Carrier individuals do not show symptoms of amoebiasis even though they remain infectious, this complicates efforts to curtail this disease. Some infectious individuals may recover naturally at the rate $\eta_{1}$ or by treatment at the rate $\tau$, while others progress to carriers at the rate $\gamma$. Screening is conducted to the general population to detect if there exist some carriers; as such, the detected carriers join infectious individuals rate, $\theta$ others may recover naturally at the rate $\eta_{2}$. Amoebiasis is fatal, so infectious individuals and carriers may die at the rate $d_{1}$ and $d_{2}$ respectively. Any individual may suffer natural death whose rate is modelled by $\mu$. It is known that amoebiasis induce temporal immunity that wanes at the rate $\phi$. Therefore, recovered individuals may join the susceptible class when they lose their immunity. Infected individuals from both states $I(t)$ and $C(t)$ shed pathogens into the environment at the rates $\epsilon_{1}$ and $\epsilon_{2}$ respectively. However, the rate of shedding by the infectious individuals, $\epsilon_{1}$, is significantly higher than that by the carrier group, $\epsilon_{2}$. Note that despite the low shedding of pathogens by the carrier group, because of its extremely long duration without showing any disease symptoms, the carrier group plays an important role in infection dynamics of amoebiasis.

The per capita growth rate of amoebiasis pathogens is denoted by $r$ while pathogens deplete naturally at a rate $\mu_{b}$ or by sanitation measures at the rate $\chi$. The growth rate of pathogens $(r)$ is assumed not to exceed its death rate $\left(\mu_{b}\right)$ (that is $r<\mu_{b}$ ). A full description of the variables and parameters to be used in the model are as shown in Table 1 and Table 2 respectively.

The flow diagram for the dynamics is given by Figure 1 .

Table 1: State variables and their description.

\begin{tabular}{ll}
\hline Variable & Description \\
\hline$S(t)$ & The number of susceptible humans at a time $t$. \\
$E(t)$ & The number of exposed humans at a time $t$. \\
$I(t)$ & The number of infectious humans at a time $t$. \\
$R(t)$ & The number of recovered (immune) humans at a time $t$. \\
$C(t)$ & The number of carriers at a time $t$. \\
$B(t)$ & The number of Amoebiasis pathogens at a time $t$. \\
\hline
\end{tabular}


Table 2: Parameters and their description.

\begin{tabular}{|c|c|c|c|}
\hline Parameter & Description & Value & Source \\
\hline$\Lambda$ & $\begin{array}{l}\text { Rate of recruitment of individuals into } \\
\text { the susceptible class }\end{array}$ & 469 Humans/year & [9] \\
\hline$\alpha_{1}$ & $\begin{array}{l}\text { Transmission rate for infectious individu- } \\
\text { als }\end{array}$ & $0.6 /$ year & [8] \\
\hline$\alpha_{2}$ & Transmission rates for carriers & $1 / 3 /$ year & [10] \\
\hline$\alpha_{3}$ & $\begin{array}{l}\text { Effective transmission rate of Amoebiasis } \\
\text { due to environment to human interaction }\end{array}$ & $0.4465 /$ year & [3] \\
\hline$\delta$ & $\begin{array}{l}\text { Incubation rate (Rate at which exposed } \\
\text { individuals, } E(t) \text { progress to either class } \\
I(t) \text { or } C(t))\end{array}$ & 1/28/day & {$[11,4]$} \\
\hline$\mu$ & Natural human mortality rate & 1/50/year & {$[10]$} \\
\hline$d_{1}$ & Disease induced death rate by $I(t)$ & $0.02 /$ year & Assumed \\
\hline$d_{2}$ & Disease induced death rate by $C(t)$ & $0.03 /$ year & Assumed \\
\hline$\eta_{1}$ & Recovery rate of infectious humans & 1/10/day & [12] \\
\hline$\eta_{2}$ & Recovery rate of carrier humans & $1 / 3 /$ year & {$[13]$} \\
\hline$\epsilon_{1}$ & $\begin{array}{l}\text { Pathogens shed rate into the water supply } \\
\text { by infectious human }\end{array}$ & 80 cells/mL/day & Assumed \\
\hline$\epsilon_{2}$ & $\begin{array}{l}\text { Pathogens shed rate into the water supply } \\
\text { by carrier humans human }\end{array}$ & 70 cells/mL/day & Assumed \\
\hline$r$ & $\begin{array}{l}\text { (Maximum) per capita growth rate of } \\
\text { Amoebiasis pathogens }\end{array}$ & $0.73 /$ day & [15] \\
\hline$\mu_{b}$ & $\begin{array}{l}\text { Mortality rate of Amoebiasis pathogens, } \\
\text { including phage degradation }\end{array}$ & $0.83 /$ day & [14] \\
\hline$\phi$ & $\begin{array}{l}\text { Wanning rate of diseased induced immu- } \\
\text { nity }\end{array}$ & 1/17/year & [16] \\
\hline$\chi$ & $\begin{array}{l}\text { Rate at which sanitation leads to death } \\
\text { of Shigella pathogens }\end{array}$ & $2 \mu_{b} /$ year & [14] \\
\hline$\gamma$ & $\begin{array}{l}\text { Natural recovery rate for infectious indi- } \\
\text { viduals }(I)\end{array}$ & $0.4 /$ year & Assumed \\
\hline$\tau$ & $\begin{array}{l}\text { Treatment rate for infectious individuals } \\
(I)\end{array}$ & $0.4 /$ year & Assumed \\
\hline$f$ & $\begin{array}{l}\text { Fraction of exposed individuals who } \\
\text { progress to infectious class }(I)\end{array}$ & 0.1 & {$[11]$} \\
\hline
\end{tabular}

From Figure 1, assumptions and model description the following system of differential 


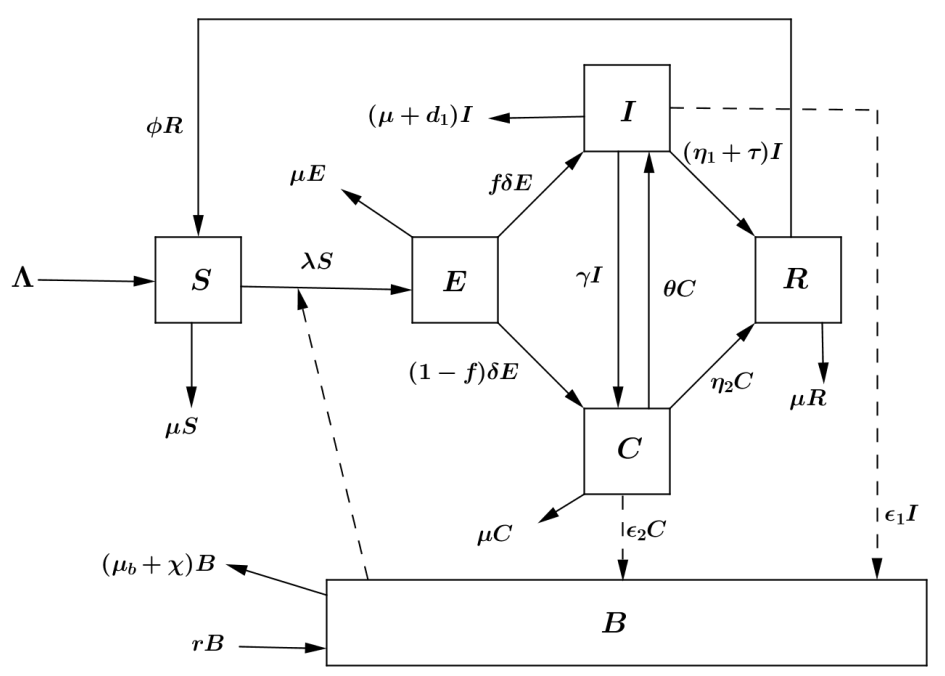

Figure 1: A flow diagram for Amoebiasis transmission dynamics.

equations are:

$$
\begin{aligned}
\frac{d S}{d t} & =\Lambda+\phi R-(\lambda+\mu) S, \\
\frac{d E}{d t} & =\lambda S-(\mu+\delta) E, \\
\frac{d I}{d t} & =f \delta E+\theta C-\left(\mu+d_{1}+\eta_{1}+\gamma+\tau\right) I, \\
\frac{d C}{d t} & =(1-f) \delta E+\gamma I-\left(\mu+d_{2}+\eta_{2}+\theta\right) C, \\
\frac{d R}{d t} & =\left(\eta_{1}+\tau\right) I+\eta_{2} C-(\mu+\phi) R, \\
\frac{d B}{d t} & =r B+\epsilon_{1} I+\epsilon_{2} C-\left(\mu_{b}+\chi\right) B .
\end{aligned}
$$

The initial conditions for the model system (2) are $S(0)>0, E(0)>0, I(0)>0$, $C(0)>0, R(0)>0, B(0)>0$.

\section{Boundedness of solutions}

The model system (2) can be partined into two parts that contains, the human population $T_{H}$ and the concentration of the pathogens in the aquatic environment $T_{B}$ such that $T_{H}=\left\{(S(t), E(t), I(t), C(t), R(t)) \in \mathbb{R}_{+}^{5}: S(t)+E(t)+I(t)+C(t)+R(t)=N(t)\right\}$ and $T_{B}=\left\{B(t) \in \mathbb{R}_{+}^{1}\right\}$ respectively. From the model system (2), the differential inequality of the susceptible population is given by

$$
\frac{d S}{d t}+\mu S \leq \Lambda+\phi R
$$


The differential inequality (3) can be solved to obtain

$$
S(t) \leq \frac{\Lambda}{\mu}+e^{-\mu t} \int_{0}^{t} \phi R(x) e^{-\mu x} d x
$$

Applying the theorem of differential inequality by Birkhoff and Rota [17] we obtain

$$
\lim _{t \rightarrow \infty} \sup S(t) \leq \frac{\Lambda}{\mu}
$$

Hence, the state variable for the susceptible population is less or equal to the ratio of the recruitment rate and the natural mortality rate. We note also that the total population is given as $N=S+E+I+C+R$. Taking the time derivative of $N$ i.e $\frac{d N}{d t}$ and substitute the equations of system (2) into the resulting expression we obtain

$$
\frac{d N}{d t}=\Lambda-\mu N-d_{1} I-d_{2} C
$$

Through appropriate procedures of solving equation (5) and using initial population as $N^{0}$ we obtain:

$$
N(t) \leq \frac{\Lambda}{\mu}-\left(\frac{\Lambda}{\mu}-N^{0}\right) e^{-\mu t}
$$

therefore,

$$
\lim _{t \rightarrow \infty} \sup N(t) \leq \frac{\Lambda}{\mu_{h}}
$$

Since $N$ is the total population, then each of the individual state variables is less or equal to $\frac{\Lambda}{\mu}$. Using equation for $B$ from the system (2), we assume that the growth rate of the pathogen in linear at a constant rate $r$. We therefore obtain a differential inequality

$$
\frac{d B}{d t}+\left(\mu_{b}+\chi-r\right) B \leq \epsilon_{1} I+\epsilon_{2} C=\left(\epsilon_{1}+\epsilon_{2}\right) \frac{\Lambda}{\mu}
$$

The solution to equation(7) can be obtained by using a suitable techniques to get

$$
B(t) \leq A e^{-\left(\mu_{b}+\chi-r\right) t}
$$

therefore,

$$
\lim _{t \rightarrow \infty} \sup B(t) \leq \frac{\left(\epsilon_{1}+\epsilon_{2}\right) \Lambda}{\mu\left(\mu_{b}+\chi-r\right)}
$$

Hence, the domain of biological significance of the system (2) is

$$
T=\left[S, E, I, C, R, B \geq 0: S+E+I+C+R \leq \frac{\Lambda}{\mu}, B \leq \frac{\left(\epsilon_{1}+\epsilon_{2}\right) \Lambda}{\mu\left(\mu_{b}+\chi-r\right)}\right] .
$$

The domain $T$ is positively invariant under the flow induced by the system (2). Therefore, the system (2) is biologically meaningful, and it is feasible to analyze the model in the domain $T$. 


\section{Existence of equilibrium solutions}

In this part, we shall establish the existence of the equilibrium. In order to determine the equilibrium points, we set right hand side of the system (2) to zero and solve the resulting system:

$$
\begin{aligned}
& 0=\Lambda+\phi R^{*}-\left(\lambda^{*}+\mu\right) S^{*}, \\
& 0=\lambda^{*} S^{*}-(\mu+\delta) E^{*}, \\
& 0=f \delta E^{*}+\theta C^{*}-\left(\mu+d_{1}+\eta_{1}+\gamma+\tau\right) I^{*}, \\
& 0=(1-f) \delta E^{*}+\gamma I-\left(\mu+d_{2}+\eta_{2}+\theta\right) C^{*}, \\
& 0=\left(\eta_{1}+\tau\right) I^{*}+\eta_{2} C^{*}-(\mu+\phi) R^{*}, \\
& 0=r B^{*}+\epsilon_{1} I^{*}+\epsilon_{2} C^{*}-\left(\mu_{b}+\chi\right) B^{*} .
\end{aligned}
$$

where

$$
\lambda^{*}=\beta_{1} I^{*}+\alpha_{2} C^{*}+\alpha_{3} B^{*} .
$$

To simplify solving of the system (10), let $w_{0}, w_{2}, w_{3}>0$ such that

$$
\begin{aligned}
& w_{0}=\mu+\delta, \\
& w_{1}=\mu+d_{1}+\eta_{1}+\gamma+\tau, \\
& w_{2}=\mu+d_{2}+\eta_{2}+\theta, \\
& w_{3}=\mu_{b}+\chi-r .
\end{aligned}
$$

Solving the system (10) we have

$$
\begin{aligned}
E^{*} & =h_{1} I^{*} \\
C^{*} & =h_{2} I^{*} \\
R^{*} & =h_{3} I^{*} \\
S^{*} & =\frac{\Lambda}{\mu}+\left(\frac{\phi h_{3}}{\mu}-\frac{(\mu+\delta) h_{1}}{\mu}\right) I^{*}, \\
B^{*} & =\left(\frac{\epsilon_{1}+\epsilon_{2} h_{2}}{w_{3}}\right) I^{*} .
\end{aligned}
$$

and

$$
\begin{aligned}
h_{1} & =\frac{w_{1} w_{2}-\theta \gamma}{f \delta w_{2}+(1-f) \delta \theta}, \\
h_{2} & =\frac{f \delta+(1-f) w_{1} \delta}{f \delta w_{2}+(1-f) \delta \theta}, \\
h_{3} & =\frac{\eta_{1}+\tau+\eta_{2} h_{2}}{f \delta w_{2}+(1-f) \delta \theta}
\end{aligned}
$$

It can be noted that

$$
\lambda^{*}=\alpha_{1} I^{*}+\alpha_{2} C^{*}+\alpha_{3} B^{*}=h_{4} I^{*}
$$

where

$$
h_{4}=\alpha_{1}+\alpha_{2} h_{2}+\frac{\alpha_{3}\left(\epsilon_{1}+\epsilon_{2} h_{2}\right)}{w_{3}}
$$


Substituting $\lambda^{*}, S^{*}$ and $E^{*}$ into second equation of the system (10), we obtain

$$
h_{4} I^{*}\left(\frac{\Lambda}{\mu}+\left(\frac{\phi h_{3}}{\mu}-\frac{(\mu+\delta) h_{1}}{\mu}\right) I^{*}\right)=(\mu+\delta) h_{1} I^{*}
$$

Equation (15) has two solutions:

$$
\begin{aligned}
I^{*} & =0, \\
I^{*} & =\frac{\Lambda}{\mu+\delta}+\frac{\phi h_{3}}{\mu+\delta}-\frac{1}{h_{4}}
\end{aligned}
$$

$I^{*}=0$ correspond to the existence of disease free whereas $I^{*}=\frac{\Lambda}{\mu+\delta}+\frac{\phi h_{3}}{\mu+\delta}-\frac{1}{h_{4}}$ correspond to the existence of the endemic equilibrium point.

Disease Free Equilibrium Point

The disease free equilibrium is obtained in the absence of infection. Thus the disease free exist when $I^{*}=0$, substituting into equation (13) we get $E^{*}=C^{*}=R^{*}=B^{*}=0$ and $S^{*}=\frac{\Lambda}{\mu}$. Hence, the disease free equilibrium is given by

$$
E_{0}=\left(S^{0}, E^{0}, I^{0}, C^{0}, R^{0}, B^{0}\right)=\left(\frac{\Lambda}{\mu}, 0,0,0,0,0\right) .
$$

\section{Endemic Equilibrium Point}

The nontrivial equilibrium point of the model system (10) is the point the endemic equilibrium point. It is obtained when: $S^{*}, E^{*}, I^{*}, C^{*}, R^{*}, B^{*}>0$. In this particular case, we obtain an endemic equilibrium point by substituting $I^{*}=\frac{\Lambda}{\mu+\delta}+\frac{\phi h_{3}}{\mu+\delta}-\frac{1}{h_{4}}>0$ into equation (13).

\section{Reproduction number and stability analysis of steady states}

Effective reproduction number denoted by $R_{e}$ is the average number of secondary infections caused by an infectious individual during its entire period of infectiousness Diekmann et al.,2009 [18]. The effective reproduction number is a crucial non-dimensional quantity in epidemiology as it sets the threshold in the study for predicting its outbreak and evaluating its control strategies. Thus, whether a disease becomes persistent or dies out in a community depends on the value of the reproduction number. Furthermore, the stability of equilibria can be analyzed using $R_{e}$. If $R_{e}<1$, every infectious individual will cause less than one secondary infection. Therefore, the disease will die out, and when $R_{e}>1$, every infectious individual will generate greater than one secondary infection, and hence the disease will invade the population. A large value of $R_{e}$ may indicate the possibility of a major outbreak of the disease. We compute the effective reproduction number, $R_{e}$, using the next generation operator approach as in work by Van den Driessche and Watmough [19]. The effective reproduction number is obtained by taking the largest (dominant) eigenvalue (spectral radius) of the matrix

$$
F V^{-1}=\left[\frac{\partial \mathcal{F}_{i}\left(E_{0}\right)}{\partial x_{j}}\right]\left[\frac{\partial \mathcal{V}_{i}\left(E_{0}\right)}{\partial x_{j}}\right]^{-1}
$$

where $\mathcal{F}_{i}$ is the rate of appearance of new infection in compartment $i, \mathcal{V}_{i}$ is the transfer of infections from compartment $i$ to another and $E_{0}$ is the disease-free equilibrium. From 
system (2), we re-write the equations with infectious classes, $E, I, C$ and $B$. This leads to the system

$$
\begin{aligned}
\frac{d E}{d t} & =\lambda S-w_{0} E, \\
\frac{d I}{d t} & =\delta E-w_{1} I, \\
\frac{d C}{d t} & =\gamma I-w_{2} C, \\
\frac{d B}{d t} & =\epsilon_{1} I+\epsilon_{2} C-w_{3} B .
\end{aligned}
$$

where $w_{0}, w_{1}, w_{2}, w_{3}$ have been defined in equation (12). From the system (19) we obtain:

$$
\begin{aligned}
\mathcal{F}_{i} & =\left[\begin{array}{c}
\alpha_{3} B S+\alpha_{1} I S+\alpha_{2} C S \\
0 \\
0 \\
0
\end{array}\right] \\
\mathcal{V}_{i} & =\left[\begin{array}{c}
w_{0} E \\
w_{1} I-\delta E \\
w_{2} C-\gamma I \\
\left(\mu_{b}+\chi-r\right) B-\epsilon_{1} I-\epsilon_{2} C
\end{array}\right]
\end{aligned}
$$

Partial derivative of $\mathcal{F}_{i}$ and $\mathcal{V}_{i}$ with respect to $E, I, C$ and $B$ evaluated at $E_{0}$ gives

$$
\begin{aligned}
& F=\left[\begin{array}{cccc}
0 & \alpha_{1} S^{0} & \alpha_{2} S^{0} & \alpha_{3} S^{0} \\
0 & 0 & 0 & 0 \\
0 & 0 & 0 & 0 \\
0 & 0 & 0 & 0
\end{array}\right] \\
& V=\left[\begin{array}{cccc}
w_{0} & 0 & 0 & 0 \\
-f \delta & w_{1} & -\theta & 0 \\
f-1 & -\gamma & w_{2} & 0 \\
0 & -\epsilon_{1} & -\epsilon_{2} & w_{3}
\end{array}\right]
\end{aligned}
$$

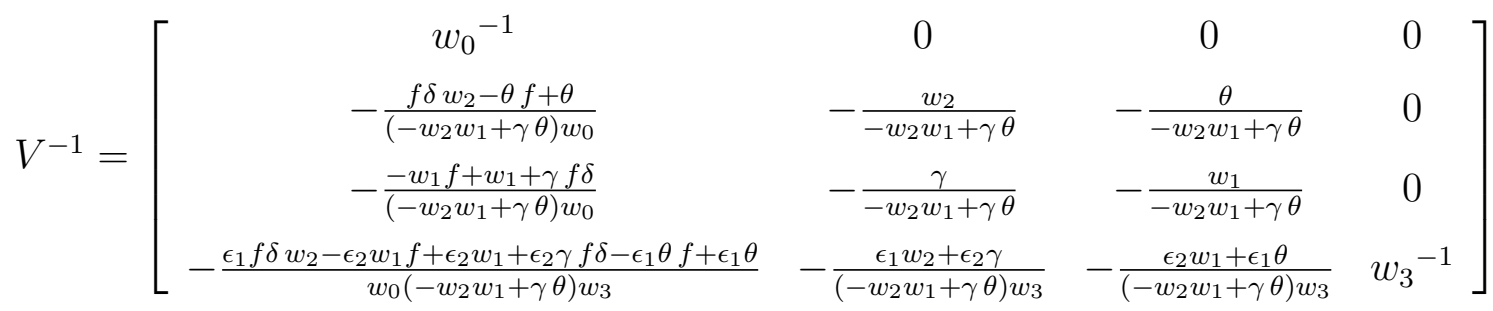

The effective reproduction number is now given by

$$
R_{e}=\rho\left(F V^{-1}\right)=R_{1}+R_{2}+R_{3}
$$


where

$$
\begin{aligned}
R_{1} & =\frac{\left(f \delta w_{2}+(1-f) \theta\right) \alpha_{1} S^{0}}{\left(w_{2} w_{1}-\gamma \theta\right) w_{0}} \\
R_{2} & =\frac{\left(w_{1}(1-f)+\gamma f \delta\right) \alpha_{2} S^{0}}{\left(w_{2} w_{1}-\gamma \theta\right) w_{0}} \\
R_{3} & =\frac{\left(\epsilon_{1} f \delta w_{2}+\epsilon_{2} \gamma f \delta+(1-f)\left(\epsilon_{1} \theta+\epsilon_{2} w_{1}\right)\right) \alpha_{3} S^{0}}{\left(w_{2} w_{1}-\gamma \theta\right) w_{0} w_{3}}
\end{aligned}
$$

while terms $w_{0}, w_{1}, w_{2}$ and $w_{3}$ have been defined in (12) whereas $S^{0}$ is defined in equation (17). Moreover, $R_{i}(i=1,2,3)$ are partial basic reproduction number induced by susceptible-to-infectious, susceptible-to-carrier transmission and environment-to-susceptible transmission, respectively.

The basic reproduction number: We deduce the basic reproduction from the effective reproduction number in equation (24) by setting the control parameters to zero: $\theta=\tau=$ $\chi=0$. Therefore, the basic reproduction number is given by

$$
R_{0}=R_{1}^{\prime}+R_{2}^{\prime}+R_{3}^{\prime}
$$

where

$$
\begin{aligned}
& R_{1}^{\prime}=R_{1}(\theta=0, \chi=0, \tau=0), \\
& R_{2}^{\prime}=R_{2}(\theta=0, \chi=0, \tau=0) \\
& R_{3}^{\prime}=R_{3}(\theta=0, \chi=0, \tau=0)
\end{aligned}
$$

each term $R_{i}^{\prime}(i=1,2,3)$ shows the contribution from infectious, carriers and environment respectively.

\section{Local stability of disease-free equilibrium}

Here we establish the local stability of disease-free equilibrium that is obtained in equation (17). This is stated in the Theorem 1 as follows:

Theorem 1. The DFE of the model system (2) is locally asymptotically stable if $R_{e}<1$ and unstable if $R_{e}>1$.

Proof. The partial differentiation of system (2) with respect to $(S, E, I, C, R, B)$ at the disease free equilibrium gives the Jacobian Matrix $J$ as

$$
J\left(E_{0}\right)=\left[\begin{array}{cccccc}
-\mu & 0 & -\alpha_{1} S_{0} & -\alpha_{2} S_{0} & \phi & -\alpha_{3} S_{0} \\
0 & -w_{0} & \alpha_{1} S_{0} & \alpha_{2} S_{0} & 0 & \alpha_{3} S_{0} \\
0 & \delta & -w_{1} & 0 & 0 & 0 \\
0 & 0 & \gamma & -w_{2} & 0 & 0 \\
0 & 0 & \eta_{1}+\tau & \eta_{2} & -w_{3} & 0 \\
0 & 0 & \epsilon_{1} & \epsilon_{2} & 0 & -w_{4}
\end{array}\right]
$$

where $w_{0}, w_{1}$ and $w_{2}$ have been defined in equation (12).

The matrix (26) has two trivial negative eigenvalues $\lambda=-\mu$ and $\lambda=-w_{3}=-\left(\mu_{b}+\chi-r\right)$. 
If we set $A_{11}=w_{0}, A_{12}=\alpha_{1} S_{0}, A_{13}=\alpha_{2} S_{0}, A_{14}=S_{0} \alpha_{3}, A_{21}=\delta, A_{22}=w_{1}, A_{32}=\gamma$, $A_{33}=w_{2}, A_{42}=\epsilon_{1}, A_{43}=\epsilon_{2}, A_{44}=\mu_{b}+\chi-r$, then the remaining $4 \times 4$ sub-matrix is given as:

$$
J_{1}\left(E_{0}\right)=\left[\begin{array}{cccc}
-A_{11} & A_{12} & A_{13} & A_{14} \\
A_{21} & -A_{22} & 0 & 0 \\
0 & A_{32} & -A_{33} & 0 \\
0 & A_{42} & A_{43} & -A_{44}
\end{array}\right]
$$

The rest of the eigenvalues are the roots of the polynomial: $\left|J_{1}\left(E_{0}\right)-\lambda\right|=0$, which is given by

$$
\lambda^{4}+c_{3} \lambda^{3}+c_{2} \lambda^{2}+c_{1} \lambda+c_{0}=0
$$

where the constants are such that

$$
\begin{aligned}
c_{3}= & A_{11}+A_{22}+A_{33}+A_{44} \\
c_{2}= & \left(A_{44} A_{33}+A_{44} A_{22}+A_{44} A_{11}+A_{33} A_{22}+A_{33} A_{11}+A_{22} A_{11}\left(1-R_{04}\right)\right) \\
c_{1}= & H_{11} H_{22} H_{44}\left(1-\left(R_{b}+R_{g}\right)\right)+H_{11} H_{33} H_{44}\left(1-\left(R_{c}+R_{d}\right)\right)+H_{11} H_{22} H_{33}\left(1-\left(R_{d}+R_{f}+R_{g}\right)\right) \\
& +H_{22} H_{33} H_{44} \\
c_{0}= & A_{44} A_{33} A_{22} A_{11}\left(1-R_{e}\right)
\end{aligned}
$$

moreover, $R_{e}$ can be split into parts

$$
R_{e}=R_{01}+R_{02}+R_{03}+R_{04},
$$

where

$$
\begin{aligned}
R_{01} & =\frac{A_{43} A_{32} A_{21} A_{14}}{A_{44} A_{33} A_{22} A_{11}}, \\
R_{02} & =\frac{A_{42} A_{21} A_{14} A_{33}}{A_{44} A_{33} A_{22} A_{11}}, \\
R_{03} & =\frac{A_{44} A_{32} A_{21} A_{13}}{A_{44} A_{33} A_{22} A_{11}}, \\
R_{04} & =\frac{A_{44} A_{33} A_{21} A_{12}}{A_{44} A_{33} A_{22} A_{11}} .
\end{aligned}
$$

To ensure that all roots of equation (28) have negative real parts, the Routh-Hurwitz stability criterion requires that

$$
c_{3}>0, c_{2}>0, c_{1}>0, c_{0}>0,
$$


and

$$
\begin{aligned}
D_{1} & =c_{3}>0 \\
D_{2} & =\left|\begin{array}{cc}
c_{3} & 1 \\
c_{1} & c_{2}
\end{array}\right|=c_{3} c_{2}-c_{1}>0 \\
D_{3} & =\left|\begin{array}{ccc}
c_{3} & 1 & 0 \\
c_{1} & c_{2} & c_{3} \\
0 & c_{0} & c_{1}
\end{array}\right|=c_{1} c_{2} c_{3}-c_{1}^{2}-c_{0} c_{3}^{2}>0 \\
D_{4} & =\left|\begin{array}{cccc}
c_{3} & 1 & 0 & 0 \\
c_{1} & c_{2} & c_{3} & 1 \\
0 & c_{0} & c_{1} & c_{2} \\
0 & 0 & 0 & c_{0}
\end{array}\right|=c_{0}\left(c_{1} c_{2} c_{3}-c_{1}^{2}-c_{0} c_{3}^{2}\right)>0
\end{aligned}
$$

It is obvious that $D_{1}=c_{3}>0$. In addition, if $R_{e}<1$, it implies that $R_{a}, R_{b}, R_{c}, R_{d}, R_{f}$, $R_{g}<1$ and hence $c_{0}, c_{1}, c_{2}>0$.

Also, $D_{2}$ can be shown to be positive as follows:

$$
\begin{aligned}
D_{2}=c_{2} c_{3}-c_{1} & =H_{11}\left(H_{22}\left(H_{44}\left(R_{b}+2\right)+H_{33}\left(R_{f}+2\right)\right)+H_{33} H_{44}\left(R_{c}+2\right)+H_{33}^{2}\left(1-R_{d}\right)+p_{1}\right) \\
& +H_{11}^{2}\left(H_{33}\left(1-R_{d}\right)+H_{22}\left(1-R_{g}\right)+H_{44}\right)+\left(H_{22}+H_{33}\right)\left(H_{22}+H_{44}\right)\left(H_{33}+H_{44}\right)
\end{aligned}
$$

where $p_{1}=H_{22}^{2}\left(1-R_{g}\right)+H_{44}^{2}$, hence $D_{2}$ is positive.

The only remaining condition to show is

$$
D_{3}=c_{1}\left(c_{2} c_{3}-c_{1}\right)-c_{0} c_{3}^{2}>0 .
$$

To prove the inequality (36), it is sufficient to establish the following two inequalities:

$$
\begin{gathered}
c_{1} c_{2} c_{3}>2 c_{1}^{2} \\
c_{1} c_{2} c_{3}>2 c_{0} c_{3}^{2}
\end{gathered}
$$

To show (37), we write $c_{2} c_{3}-2 c_{1}$ into the sum of the following parts:

$$
\begin{aligned}
c_{2} c_{3}-2 c_{1} & =2 H_{22} H_{44} H_{11} R_{b}+2 H_{33} H_{44} H_{11} R_{c}+H_{33} H_{11}^{2}\left(1-R_{d}\right)+H_{33}^{2} H_{11}\left(1-R_{d}\right) \\
& +H_{22} H_{33} H_{11} R_{d}+H_{33} H_{44} H_{11} R_{d}+2 H_{22} H_{33} H_{11} R_{f}+H_{22} H_{11}^{2}\left(1-R_{g}\right) \\
& +H_{22}^{2} H_{11}\left(1-R_{g}\right)+H_{22} H_{33} H_{11} R_{g}+H_{22} H_{44} H_{11} R_{g}+H_{44} H_{11}^{2}+H_{22}^{2} H_{11} \\
& +H_{44}^{2} H_{11}+H_{22} H_{33} H_{11}+H_{22} H_{44} H_{11}+H_{33} H_{44} H_{11}+H_{22} H_{33}^{2}+H_{22} H_{44}^{2} \\
& +H_{33} H_{44}^{2}+H_{22}^{2} H_{33}+H_{22}^{2} H_{44}+H_{33}^{2} H_{44}+H_{22} H_{33} H_{44}
\end{aligned}
$$

Similarly, to show (38), we write $c_{1} c_{2}-2 c_{0} c_{3}$ into the sum of parts as follows:

$$
\begin{aligned}
c_{1} c_{2}-2 c_{0} c_{3} & =H_{11} H_{22} H_{33}^{2} H_{44}\left(\left(1-R_{d}\right)+R_{c}+R_{f}+R_{g}+2 R_{a}+2 R_{b}\right) \\
& +H_{11} H_{22}^{2} H_{33} H_{44}\left(\left(1-R_{g}\right)+R_{b}+R_{d}+R_{f}+2 R_{a}+2 R_{c}\right) \\
& +H_{11}^{2} H_{22} H_{33} H_{44}\left(1-\left(R_{d}+R_{g}\right)+2 R_{a}+R_{b}+R_{c}+R_{f}+R_{b} R_{d}+2 R_{d} R_{g}+R_{c} R_{g}\right) \\
& +H_{11}^{2} H_{22}^{2} H_{33}\left(1-\left(R_{d}+R_{f}+R_{g}\right)+R_{d} R_{g}+R_{f} R_{g}+R_{g}^{2}\right) \\
& +H_{11} H_{22}^{2} H_{33}^{2}\left(1-R_{d}-R_{f}-R_{g}\right)+H_{11}^{2} H_{22} H_{33}^{2}\left(1-R_{d}\right)\left(1-R_{d}-R_{f}-R_{g}\right) \\
& +H_{11} H_{22}^{2} H_{44}^{2}\left(1-R_{b}-R_{g}\right)+H_{11}^{2} H_{22} H_{44}^{2}\left(1-R_{b}-R_{g}\right) \\
& +H_{11}^{2} H_{22}^{2} H_{44}\left(1-R_{g}\right)\left(1-R_{b}-R_{g}\right)+H_{11} H_{33}^{2} H_{44}^{2}\left(1-R_{c}-R_{d}\right) \\
& +H_{11}^{2} H_{33}^{2} H_{44}\left(1-R_{d}\right)\left(1-R_{c}-R_{d}\right)+H_{11}^{2} H_{33} H_{44}^{2}\left(1-R_{c}-R_{d}\right)+H_{22} H_{33}^{2} H_{44}^{2} \\
& +H_{22}^{2} H_{33}^{2} H_{44}+H_{11} H_{22} H_{33} H_{44}^{2}\left(1+R_{b}+R_{c}+R_{d}+R_{g}+2 R_{a}+2 R_{f}\right) \\
& +H_{22}^{2} H_{33} H_{44}^{2}
\end{aligned}
$$


It can be noted that if $R_{e}<1$, then each $R_{a}, R_{b}, R_{c}, R_{d}, R_{f}, R_{g}<1$ and therefore $c_{1} c_{2}-$ $2 c_{0} c_{3}>0$ and $c_{2} c_{3}-2 c_{1}>0$. With these results, it can be concluded that equations (37) and (38) hold and so does the condition (36). Moreover, the proof for condition $D_{4}$ can be established from the fact that $D_{4}=c_{0} D_{3}$. Fortunately, we have already proved that $D_{3}>0$, therefore it is clear that $D_{4}=c_{0} D_{3}>0$. Hence, all conditions of Routh-Hurwitz for this case (equations (34) and (35)) are satisfied then the disease free equilibrium $E_{0}$ is locally asymptotically stable whenever $R_{e}<1$.

\section{Global stability of disease free equilibrium point}

We have the following result on the global stability of the disease-free equilibrium.

Theorem 2. If $R_{e}<1$, the disease free equilibrium point is globally asymptotically stable and unstable if $R_{e}>1$.

Proof. By the comparison theorem, the rate of change of the variables representing the infected components of model system (2) can be re-written as,

$$
\left(\begin{array}{c}
\frac{d E}{d t} \\
\frac{d I}{d t} \\
\frac{d C}{d t} \\
\frac{d B}{d t}
\end{array}\right)=(F-V)\left(\begin{array}{c}
E \\
I \\
C \\
B
\end{array}\right)-\left(\begin{array}{cccc}
0 & \alpha_{1} S^{0} & \alpha_{2} S^{0} & \alpha_{3} S^{0} \\
0 & 0 & 0 & 0 \\
0 & 0 & 0 & 0 \\
0 & 0 & 0 & 0
\end{array}\right)\left(\begin{array}{c}
E \\
I \\
C \\
B
\end{array}\right)
$$

implying that

$$
\left(\begin{array}{c}
\frac{d E}{d t} \\
\frac{d I}{d t} \\
\frac{d C}{d t} \\
\frac{d B}{d t}
\end{array}\right) \leq(F-V)\left(\begin{array}{c}
E \\
I \\
C \\
B
\end{array}\right)
$$

where $F$ and $V$ are Jacobian matrices as in (22) and (23). Since the eigenvalues of the matrix $(F-V)$ have negative real parts (this comes from the stability results in Theorem 1 , then system (2) is stable whenever $R_{e}<1$. So $(S, E, I, C, B, R) \rightarrow\left(S^{0}, 0,0,0,0,0\right)$ and $S \rightarrow S_{0}$ as $t \rightarrow \infty$. By the comparison theorem (see Shaban 2015 [20]) $(S, E, I, C, B, R) \rightarrow E_{0}$ as $t \rightarrow \infty$. Therefore, $E_{0}$ is globally asymptotically stable whenever $R_{e}<1$.

\section{Results and discussions}

In this section, our model (2) was solved numerically via the Rungekuta fourth-order method, and it was implemented in MATLAB software. Various graphical representations are presented and discussed with the aim of validating some analytical findings presented previously. So long, most of the parameters were not easily available; we employed those obtained from the Literature survey, while others were assumed for the purpose of illustration. To aid simulations, baseline values of parameters from Table 2 presented before were used. Initial values for sub-populations are given as follows: $S(0)=60, E(0)=40, I(0)=$ $20, C(0)=90, R(0)=20, B(0)=500$. 


\section{Effects of screening intervention}

Here we discuss how screening intervention can be useful to mitigate amoebiasis disease. It can be seen from Figure 2(a) that an increase in screening rate $\theta$ has an effect of decreasing carriers; the screened individuals join the infectious class where they are finally treated. Similarly, it can be noted from Figure 2(c) as a result of more screening then, amoebiasis is greatly minimized since screened carriers receive medical attention, and hence contamination of the environment becomes minimized. Moreover, it can be noted from Figure 2(b) that too much screening implies increasing the recovered population since most people identified ill during this process are well attended.

\section{Effects of treatment intervention}

One can be seen from Figure 3(a) that with an increased treatment rate, $\tau$, there is a corresponding decrease in infectious individuals, $I$. On the contrary, it can be visualized that adequate treatment implies more individuals recover, see Figure 3(b). It can be witnessed from Figure 3(c) that an increase in treatment rate $\tau$ tends to reduce the pathogens related to this disease greatly.

\section{Effects of sanitation intervention}

The results from Figure 4 show that with an increase in sanitation effort(especially water chlorination, boiling drinking water) at rate $\chi$, there is a corresponding decrease in the number of pathogens in water. This is because most of them will not survive when subjected to adequate dosage of chlorine in municipal water. On the other hand, an investment in sanitary activity such as proper sewage disposal personal hygienic practices can increase the chance of eliminating amoebiasis from the community.

\section{Sensitivity Analysis}

The sensitivity analysis explores how the model output is affected by the variability in the model inputs. It's used to establish how sensitive a model is to changes in its structure and parameter values. It aids in developing model confidence by evaluating the uncertainties commonly linked to model parameters. Importantly, it permits us to estimate the relative changes whenever a parameter is modified. It is frequently used to assess the model's responsiveness to parameter values. As a result, we use it to identify parameters that significantly impact the model that intervention initiatives should prioritize. Sensitivity for the model (2) will be determined via the effective reproduction number $R_{e}$ given by the equation (24). An analytical expression for sensitivity to each parameter can be derived using the normalized forward sensitivity index as by Chitnis [21] as follows:

$$
\begin{aligned}
& \Gamma_{\Lambda}^{R_{e}}=\frac{\partial R_{e}}{\partial \Lambda} \times \frac{\Lambda}{R_{e}}=+1 \\
& \Gamma_{\mu}^{R_{e}}=\frac{\partial R_{e}}{\partial \mu} \times \frac{\mu}{R_{e}}=-1.9604
\end{aligned}
$$




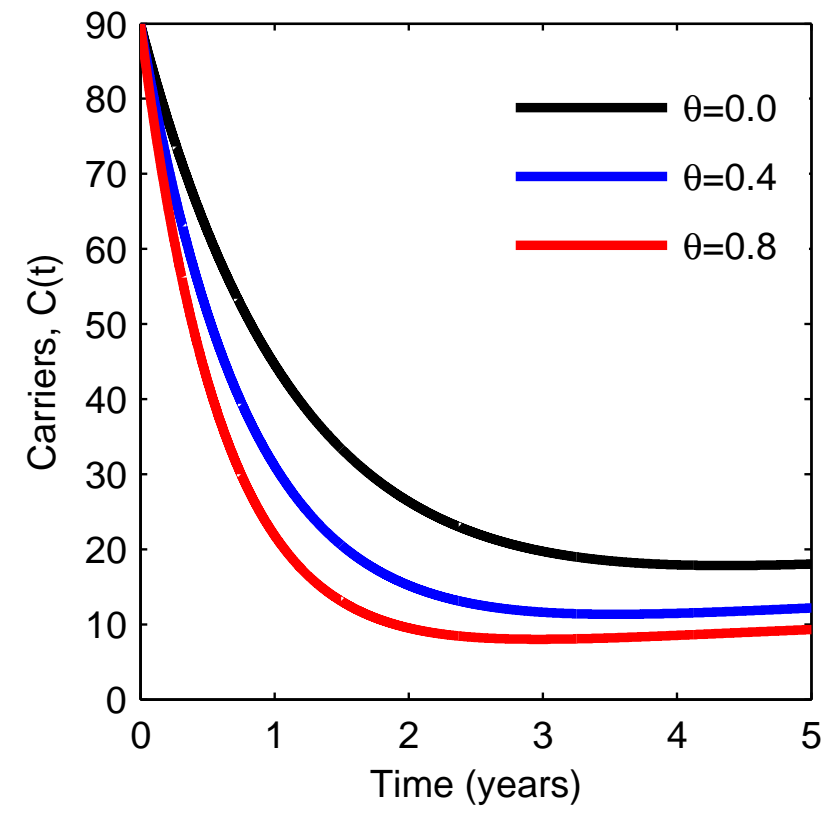

(a)

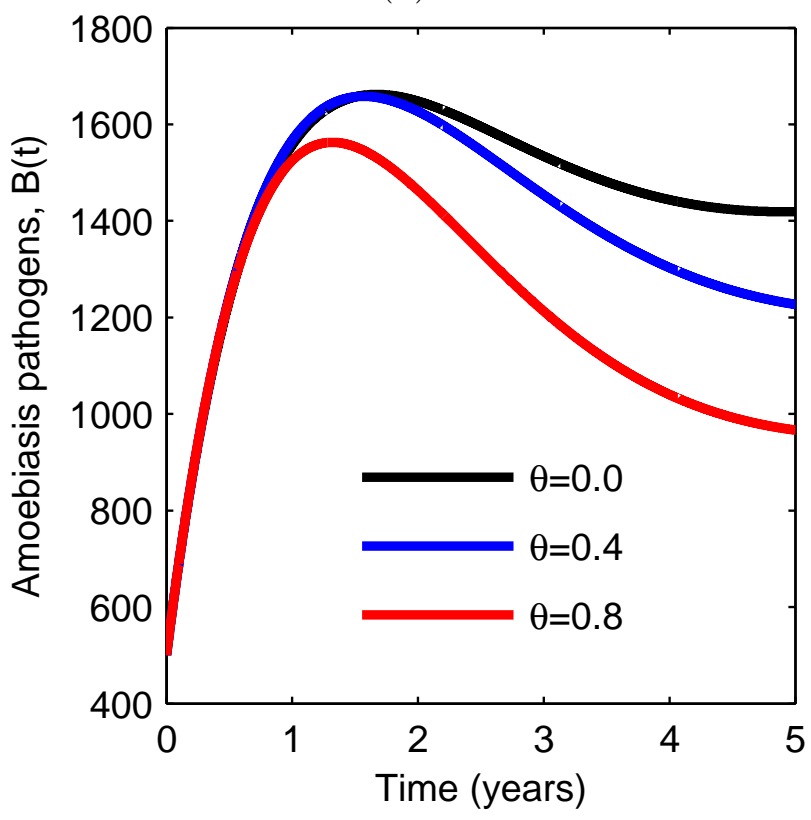

(c)

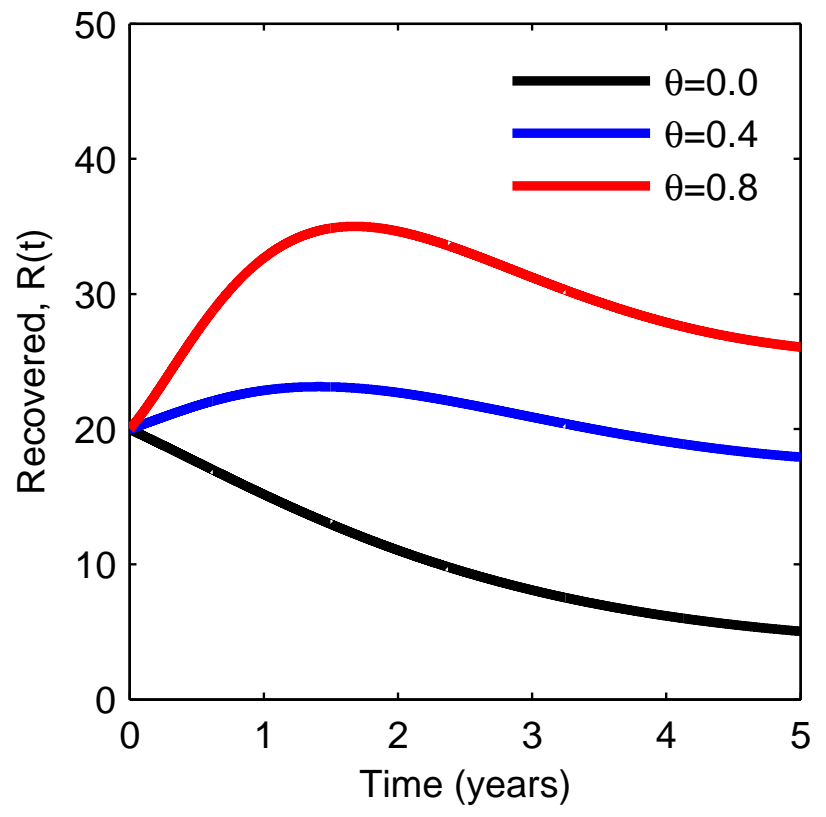

(b)

Figure 2: (a)-(c) show the effects of screening on different epidemiological classes of Amoebiasis transmission dynamics. 


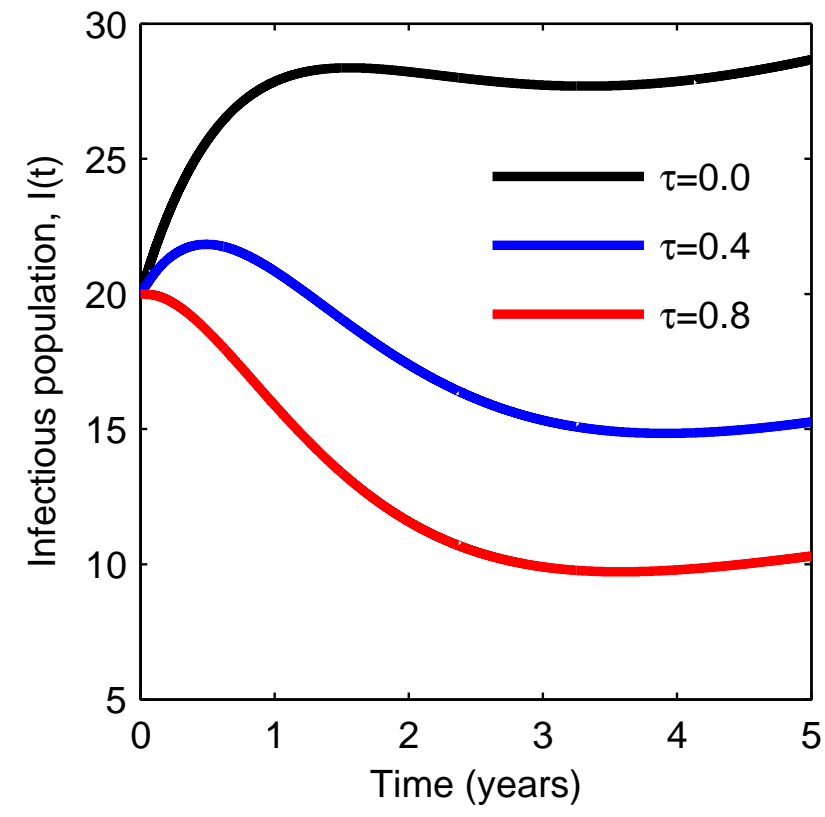

(a)

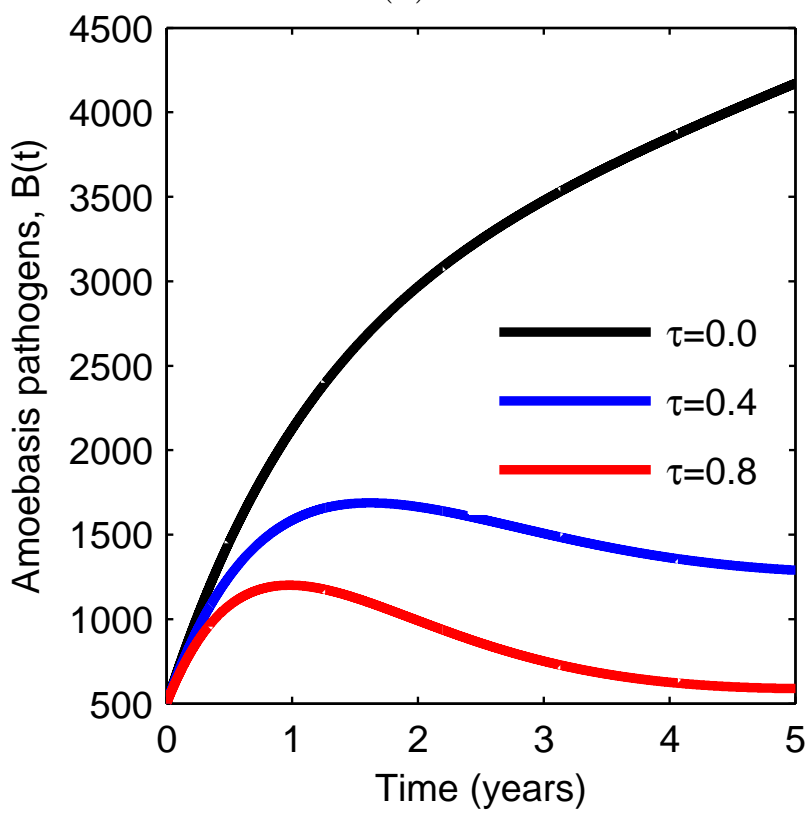

(c)

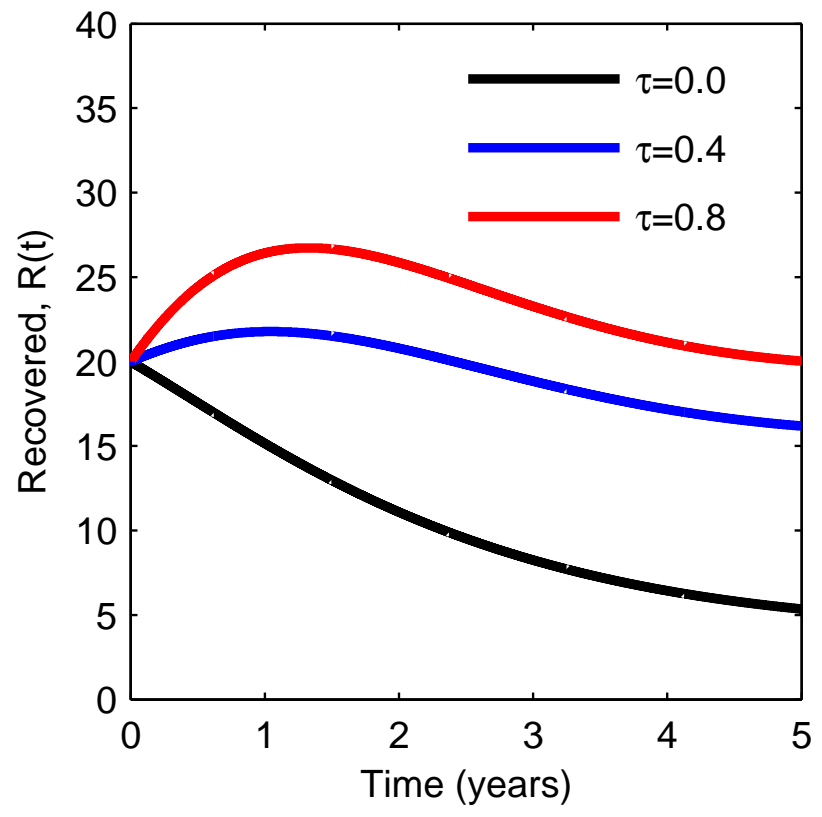

(b)

Figure 3: (a)-(c) show the effects of treatment rate $\tau$, on different epidemiological classes of Amoebiasis transmission dynamics. 


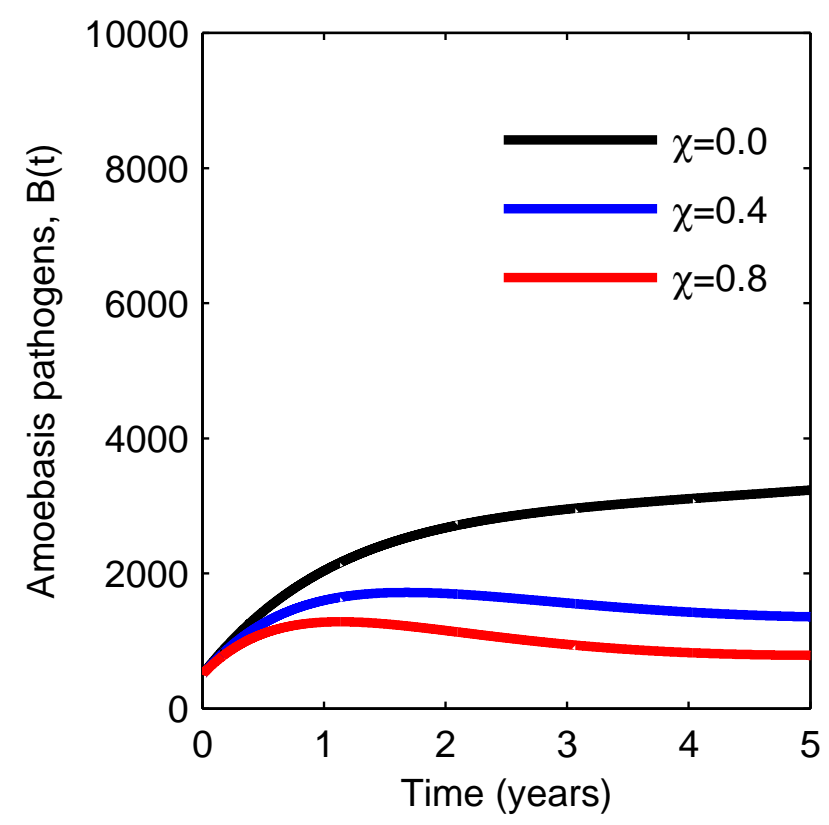

Figure 4: Effects of variation of rate of sanitation $(\chi)$ on pathogens $(B(t))$ population in the environment.

Similarly, the remaining sensitivity index values across all values employed in the expression (24) can be derived. The sensitivity coefficients of $R_{e}$ be seen in Table 3.

Table 3: Parameters and their Sensitivity Indices.

\begin{tabular}{llll}
\hline Parameter & Sensitivity Index & Parameter & Sensitivity Index \\
\hline$\Lambda$ & +1.0000 & $d_{2}$ & -0.0364 \\
$\alpha_{1}$ & +0.0134 & $\eta_{1}$ & -0.0416 \\
$\alpha_{2}$ & +0.0068 & $\eta_{2}$ & -0.0364 \\
$\alpha_{3}$ & +0.9799 & $\epsilon_{1}$ & +0.5855 \\
$\gamma$ & -0.0192 & $\epsilon_{2}$ & +0.3943 \\
$\delta$ & -0.2883 & $r$ & +0.1633 \\
$\mu$ & -1.9958 & $\tau$ & -0.2082 \\
$\mu_{b}$ & -0.6532 & $\theta$ & +0.19395 \\
$\chi$ & -0.4899 & $f$ & -0.0985 \\
$d_{1}$ & -0.1561 & & \\
\hline
\end{tabular}

From Table 3, one for instance can get $\Gamma_{\mu}^{R_{e}}=-1.9958$ which implies that increasing $\mu$ results in a reduction in $R_{e}$. Likewise, since $\mu$ and $R_{e}$ are inversely proportional, a decline in $\mu$ will lead to an increase in $R_{e}$. It can also be seen that $d_{1}, d_{2}, \eta_{1}, \eta_{2}, \mu_{b}, \gamma, \tau$ and $\chi$ are all negatives, suggesting that they too are all inversely related to $R_{e}$. Thus, any increase (decrease) of such parameters, will cause $R_{e}$ to decrease (increase).

On the contrary, $\Gamma_{\Lambda}^{R_{e}}=+1$ shows an increment in $\Lambda$ will result an increase in $R_{e}$. Moreover, as $\Lambda$ and $R_{e}$ are directly related, a decline in $\Lambda$ will result in a reduction in $R_{e}$. It's also worth noting that $\alpha_{1}, \alpha_{2}, \alpha_{3}, \epsilon_{1}, \epsilon_{2}, \theta$ or $r>0$ are all directly related to $R_{e}$. This entails that rising (diminishing) any of these values will produce a rise (fall) in $R_{e}$.

This implies that an increase(decrease) in any of these parameters will cause a rise (decline) in $R_{e}$. These parameters can be arranged in order of magnitude of the highest to the lowest as follows: $\mu, \Lambda, \alpha_{3}, \mu_{b}, \epsilon_{1}, \delta, d_{1}, \chi, \gamma, \eta_{1}, \epsilon_{2}, d_{2}, \alpha_{1}, \eta_{2}$ and $\alpha_{2}$. It can be seen that the effective reproduction number $R_{e}$ decreases with an increase in the parameters 
catering for control measures $(\chi, \tau)$. This suggests that these parameters are suitably considered control interventions and, therefore, to limit the spread of this disease, a suitable combination of sanitation and treatment can alleviate this epidemic. On the other hand, the results imply that contacting with contaminated matter from the environment $\left(\alpha_{3}\right)$ has proved to contribute more than human-to-human contacts $\left(\alpha_{1}\right.$ or $\left.\alpha_{2}\right)$. This has some important implications for the general public as well as policymakers. Governments must ensure that a large population has access to proper sanitary services such as potable water, toilets, adequate sewage disposal, and the like. Such effort will aid not only to minimize the number of infections from these diseases and other diarrhoea diseases.

\section{Conclusions}

In this work, we are devoted to developing a deterministic mathematical model for transmission dynamics of amoebiasis that accounts for both direct and indirect transmission, and the model has incorporated three interventions which are treatment, screening, and sanitation. The effective reproduction number was computed using the next generation matrix approach from which stability of the equilibrium was established. It was proved that the Disease Free Equilibrium (DFE) and the Endemic Equilibrium points (EE) are locally asymptotically stable (l.a.s) when $R_{e}<1$ and stable otherwise.

Numerical results show that intervention strategies such as treatment, sanitation, and screening can slow down the spread of this disease if they are implemented regularly in the community.

In addition, sensitivity analysis was conducted to determine which parameter(s) plays a vital role in disease dynamics, which could need more attention in the control efforts. It was found that investing more in implementing sanitation and treatment control measures has the effect of minimizing the number of amoebiasis. Moreover, it was noted that indirect transmission seems to contribute more to infections than direct transmission.

This work may be beneficial to a large public as it has tried to emphasize how this disease can be contacted, prevented, and treated. Moreover, the findings from this study show the benefits of incorporating interventions to control amoebiasis. In general, our model may aid policymakers in planning better on how to control this disease.

\section{Availability of data}

All the data and materials that support the findings of this study are included in the paper.

\section{Declaration of interest}

The authors declare that they have no competing interests.

\section{Funding}

This work did not receive any financial grants. 


\section{Authors' contributions}

SE: Conceptualization, Methodology, Software, Data curation, Writing-Original draft preparation. GE: Software, Validation, Writing-Reviewing and Editing, Visualization, Investigation. All authors read and approved the final manuscript.

\section{Acknowledgments}

The SEM would like to appreciate the support received from the University of Dodoma. At the same time, GEM is thankful to the Tanzania Institute of Accountancy for the support that enabled this study's completion.

\section{References}

[1] Haque, R., Ali, I.M. and Petri Jr, W.A: Prevalence and immune response to Entamoeba histolytica infection in preschool children in Bangladesh. The American journal of tropical medicine and hygiene. 60(6),1031-1034(1999).

[2] Clark, C.G. and Diamond, L.S: The Laredo strain and other:Entamoeba histolyticalike'amoebae are Entamoeba moshkovskii. Molecular and biochemical parasitology, 46(1), 11-18(1991).

[3] Stauffer, W. and Ravdin, J.I: Entamoeba histolytica: an update. Current opinion in infectious diseases, 16(5), 479-485(2003).

[4] Ximnez, C., Morn, P., Rojas, L., Valadez, A. and Gmez, A: Reassessment of the epidemiology of amebiasis: state of the art. Infection, Genetics and Evolution, 9(6), pp.1023-1032(2009)

[5] Fathi, A.M., Bahnass, M.M. and Elshahawy, I.S.: Short Communication Seroprevalence of amoebiasis in Najran Saudi Arabia. Tropical Biomedicine, 34(3),732$740(2017)$.

[6] CC, Sobin, Snehanshu Saha, Vaskar Raychoudhury, Hategekimana Fidele, and Sumana Sinha: CISER: An Amoebiasis inspired Model for Epidemic Message Propagation in DTN. arXiv preprint arXiv:1608.07670 (2016).

[7] Hategekimana, F., Saha, S. and Chaturvedi, A: Amoebiasis Transmission and Life cycle: A continuous state description by virtue of existence and uniqueness. Global Journal of Pure and Applied Mathematics, 12(1),375-390 (2016)

[8] Hategekimana, F., Saha, S. and Chaturvedi, A: Dynamics of amoebiasis transmission: stability and sensitivity analysis. Mathematics, 5(4), 58(2017)

[9] Berhe, Hailay Weldegiorgis, Oluwole Daniel Makinde, and David Mwangi Theuri. :Parameter estimation and sensitivity analysis of dysentery diarrhea epidemic model. Journal of Applied Mathematics 2019 (2019).

[10] Keeling, Matt J., and Pejman Rohani: Modeling infectious diseases in humans and animals. Princeton university press, 2011. 
[11] Samie, A., A. ElBakri, and Ra'ed AbuOdeh : Amoebiasis in the tropics: epidemiology and pathogenesis. Current topics in tropical medicine 201 (2012).

[12] Petri W.A.Jr. and Singh U: Diagnosis and Management of Amebiasis. Clinical Infectious Diseases, 1117-1125(1999).

[13] Stauffer W., Abd-Alla M. and Ravdin J.I: Prevalence and Incidence of Entamoeba histolytica Infection in 608 South Africa and Egypt. Archives of Medical Research, 372, 265-268(2006).

[14] Mwasa, A. and Tchuenche, J.M: Mathematical analysis of a cholera model with public health interventions.Biosystems, 105(3), 190-200(2011).

[15] Edward, Stephen, Eunice Mureithi, and Nyimvua Shaban: Shigellosis dynamics: modelling the effects of treatment, sanitation, and education in the presence of carriers International Journal of Mathematics and Mathematical Sciences 2020 (2020).

[16] Abd-Alla, M.D., Jackson, T.F., Rogers, T., Reddy, S. and Ravdin, J.I: Mucosal immunity to asymptomatic Entamoeba histolytica and Entamoeba dispar infection is associated with a peak intestinal anti-lectin immunoglobulin A antibody response. Infection and immunity, 74(7), 3897-3903(2006).

[17] Birkhoff, G., and G. C. Rota: Ordinary Differential Equation, Ginn. and Co., Boston,.[18] May RM (2001). Stability and Complexity in Model Ecosystems, 1982.

[18] Diekmann, O., Heesterbeek, J.A.P. and Roberts, M.G: The construction of nextgeneration matrices for compartmental epidemic models. Journal of the Royal Society Interface, 7 (47), 873-885(2009).

[19] Driessche, P.V.D. and Watmough, J: Further notes on the basic reproduction number. In Mathematical Epidemiology, 159-178(2003). Springer, Berlin, Heidelberg, 2008.

[20] Shaban, N: Modelling the Effects of Public Health Education in the Spread of Hepatitis B Disease , Applied Mathematical Sciences, 9(80),3967 -3981(2015).

[21] Chitnis, N., Hyman, J.M. and Cushing, J.M: Determining important parameters in the spread of malaria through the sensitivity analysis of a mathematical model. Bull. Math.Biology, 70(5),1272(2008).https ://doi.org/10.1007/s1153 8-008-9299-0. 